\title{
LA MÉDIATION SCIENTIFIQUE ET TECHNIQUE, ENTRE VULGARISATION ET ESPACE PUBLIC
}

- publié dans revue Quaderni, La science dans la cité, Numéro spécial N46 2002.

\author{
Paul Rasse ${ }^{1}$ \\ Université de Nice - Sophia Antipolis, Laboratoire I3M.
}

Mots clefs: vulgarisation, communication scientifique, espace public, controverses environnementales, musée de sciences, musées d'histoire naturelle, muséologie.

Résumé Nous utilisons le paradigme d'espace public pour développer un point de vue l'historique sur les questions de vulgarisation et de communication scientifique, pour en cerner les enjeux, puis d'ouvrir des pistes d'évolution possible aux musées et autres lieux de communication scientifique et technique.

L'expression "vulgarisation de la science" apparaît au XIXe siècle pour désigner le fait de diffuser les connaissances savantes en les mettant à la portée du grand public. Le terme vulgaire, du latin vulgus, concernait jusque-là la foule, qualifiant ce qui est ordinaire, général et commun; il prend sa tournure péjorative au fur et à mesure que s'affirment les valeurs bourgeoises, pour désigner en contre point les comportements populaires ${ }^{2}$. Dans le même temps, la vulgarisation se substitue progressivement à l'expression de " familiarisation de la science " qui datait, elle, du début du siècle des lumières ${ }^{3}$. Aujourd'hui on préfère parler de "communication scientifique et technique". Entre ces trois termes se devinent différents rapports que les profanes, le peuple, la société civile peuvent nouer avec la science et la technique. Au risque de quelques simplifications et sans prétendre à l'exhaustivité d'un travail d'historien, le paradigme d'espace public nous permettra, et de développer un point de vue l'historique sur ces questions et de cerner les enjeux qu'elles recouvrent, puis d'ouvrir des pistes d'évolution possible aux musées et autres lieux de communication scientifique et technique.

\section{1 - HABERMAS ET L'ESPACE PUBLIC}

Le concept d'espace public développé par Habermas aura bientôt 35 ans, et connut un tel succès que l'on est en droit de se demander, à l'instar de Neveu, si la surexploitation du filon n'aurait pas conduit à faire des matrices analytiques " une forme d'impensé, de doxa " où "les

\footnotetext{
${ }^{1}$ Paul Rasse

Professeur des universités en Sciences de l'information et de la communication - Université de Nice - Sophia Antipolis,

Directeur du Laboratoire I3M (information, milieux, médias, médiation) en sciences de l'information et de la communication.

Il vient de publier: Paul Rasse, La rencontre des mondes, Diversité culturelle et communication, éditons Armand Colin, 2006.

2 Le Robert, Dictionnaire historique de la langue française, 1999

3 Degognet François, "Historique et principe de la vulgarisation", in dictionnaire critique de la communication, sous la direction de Lucien Sfez, p.1429.

Voir aussi (phénoménotechnie, communication, popularisation)

Voir aussi Targuieff Pierre-André, “Du progrès", librio, 2001.
} 
riches analyses d'Habermas sont réduites à quelques énoncés sommaires "4.. Pour ces raisons, il nous a semblé indispensable de commencer par opérer un retour sur son travail de façon à en dégager les principes structurants, en montrant en quoi ils pouvaient servir à une analyse du champ culturel contemporain. Habermas le dit et le redit (en préface de sa première et de ses dernières éditions) "Je m'étais fixé pour objectif de déplier le type idéal de la sphère bourgeoise à partir du contexte historique propre au développement anglais, allemand et français du XVIIIe et du début du XIXè, en stylisant certains traits caractéristiques d'une réalité sociale beaucoup plus complexe ". Mettons-nous d'accord. Il s'agit de construire un idéal type dont l'intérêt dépend moins de sa réalité historique, que de sa pertinence heuristique, c'est-à-dire de la rigueur et de la logique de sa construction, autant que de ses capacités à l'intelligibilité du monde. Nous nous proposons donc, dans un premier temps, de mettre en évidence l'architecture du concept d'espace public, avant de l'utiliser comme grille d'analyse pour porter un regard nouveau sur les transformations de l'exposition scientifique et technique au plan de sa relation au public.

\section{La sphère des personnes privées rassemblées en un public}

Habermas étudie comment, à partir du XVIIe, et plus particulièrement du XVIIIe, la bourgeoisie parvient à s'affranchir de la domination culturelle et idéologique, de l'aristocratie et de l'église. Chemin faisant, elle s'initie à l'usage public du raisonnement, elle se met à penser librement, non seulement à nourrir sa réflexion, mais à mettre en débat la culture dont elle est imprégnée depuis des siècles, pour finalement en venir à mettre en question la légitimité du pouvoir en place et le bousculer.

Habermas insiste, l'espace public devient possible à partir du moment où l'urbanisation se développe et où émerge dans la bourgeoisie citadine la notion d'espace privé. " C'est en effet, souligne Wolton, la redéfinition du privé qui permet, en contrepoint, à l'espace public de se dessiner et de s'affirmer" ". L'espace public " bourgeois" se constitue au fur et à mesure que la figure traditionnelle de la communauté rurale et artisanale traditionnelle s'estompe au profit de l'individu, sujet sensible doué de raison et capable de libre-arbitre ${ }^{7}$. La sphère domestique, privée, bourgeoise, devient le lieu de l'intimité familiale, la personne se retire dans sa chambre, lit, écrit des lettres introspectives et sensibles, pense, fait usage de son jugement.

Une nouvelle pièce occupe une place centrale dans l'agencement de la maison, le salon. Pour Habermas, il est le premier élément de transition vers la constitution d'un espace public, car le salon est dévolu à la société. Une fraction de l'aristocratie est installée en ville, loin de la cour de Versailles, et la grande bourgeoisie prend l'habitude d'y recevoir ses amis, mais aussi des artistes, des philosophes des savants. C'est là que l'individu fait l'apprentissage de la parole publique.

Le modèle de sociabilité des salons conduit à une certaine indépendance vis-à-vis de la Cour, pour en faire un lieu d'échange entre les classes sociales, avec toutefois un formalisme et un

4 Neveu Erik, Les sciences sociales face à l'espace public, les sciences sociales dans l'espace public, in L'espace public et l'emprise de la communication, sous la direction d'Isabelle Paillard, Ed. Ellug, 1995 , p.40 et 44 .

5 Habermas Jürgen, L'espace public, trente ans après, Quaderni $\mathrm{N}^{\circ} 18$, automne 1992, p.162.

6 Wolton Dominique, Penser la communication, Ed. Flammarion, 1997, p.381. et in internet et après, Ed. Flammarion, 2000, p.222

7 Walder Prado J. Plinio, Le partage de la sensibilité, Espaces publics, traditions et communautés, Hermès $\mathrm{N}^{\circ} 10$, Ed CNRS , 1992, p.71. 
maniérisme langagier qui étouffent la conversation libératrice ${ }^{8}$. Certes, les précieuses invitent écrivains, artistes et savants à partager leurs libations, mais elles imposent le ton; le salon est encore lieu de plaisir galant, plus que de discussion savante. Ce n'est que plus tard, sous la Régence de Philippe d'Orléans, que la Cour perd sa position dominante au sein de l'espace public, lorsque l'esprit et l'intelligence se libèrent de l'autorité de leur hôte noble et parviennent à cette autonomie qui transformera " la conversation en critique et les bons mots en arguments" ". Notons que sous Louis XVI, les réunions de salon sont parfois appelées Musées; Rétif de la Bretonne l'écrit à propos des déjeuners que M. de la Reynière fils donne dans le salon de son hôtel particulier. "On faisait des lectures de manuscrits, les poètes récitaient leurs vers, les dramatistes déclamaient leurs pièces et en pressentaient l'effet. C'étaient de vrais musées "10. Le terme désigne aussi parfois, à cette époque, des sociétés savantes dont la dynamique interne s'apparente à celle des salons, à la différence près que cette fois, leur existence et leur programmation ne dépendent plus de la seule personne, le maître des lieux, qui invite, mais des usagers en position d'avoir un regard critique sur ce qui leur est donné à entendre et à voir. Ces derniers font appel à des financements privés, à des mécènes qui forment leur conseil, ou plus anonyme encore, à des souscripteurs (on compte 650 souscripteurs en 1784 pour le fameux Musée de Monsieur, que le guide Thierry définit comme une société de savants ou de ceux qui veulent le devenir). Appelées encore clubs ou salons, les sociétés savantes appointent des conférenciers, disposent de salles de réunions, de librairies et même parfois de salles d'exposition dans lesquelles étaient présentés de nombreux objets de curiosité, d'art ou de sciences ${ }^{11}$.

Les premiers cabinets de curiosité comme lieu de recherche, de production et d'accumulation de connaissances savantes, relèvent du même esprit. Alors qu'ils étaient généralement installés dans le sein des palais princiers ${ }^{12}$, au siècle des lumières, ils sont de moins en moins l'exclusive de l'aristocratie et se multiplient un peu partout à l'initiative de savants, ou de notables issus de la bourgeoisie. À la différence des bibliothèques qui poussent à l'érudition solitaire et silencieuse, les cabinets de curiosité sont des lieux de sociabilité cultivée. Ils participent à l'émergence d'un espace public scientifique mettant en relation savants et érudits d'origines sociales et géographiques différentes. D'abord, parce que le prestige et la rareté fait de la collection un moyen de reconnaissance sociale qui permet à la bourgeoisie montante de fréquenter l'aristocratie, de se rencontrer, d'échanger des pièces autant que des points de vue ${ }^{13}$. Des guides comme le "Guide des amateurs étrangers voyageurs à Paris" de Thierry dirigent les passionnés vers les cabinets de curiosité les plus intéressants et mentionnent les meilleures $\operatorname{cours}^{14}$. Et les savants voyagent de l'un à l'autre dans toute l'Europe. Partout, en province notamment, règne de l'obscurantisme, des dogmatismes théologiques et de la sorcellerie, les cabinets de curiosité deviennent des lieux de regroupement de la bourgeoisie locale, d'échanges et de débats entre amateurs éclairés.

8 Von der Heyden Rynsch Verena, Salon Européens, Ed. Gallimard 1993, p.41.

9 Habermas, op. cit., p. 42.

10 De la Bretonne Retif, Monsieur Nicolas, neuvième époque 1789, t. II p. 365. Cité par Poulot Dominique, Musée, Nation, patrimoine, 1789 - 1815, Ed NRF Gallimard, 1997, p 94.

11 Poulot Dominique, Musée, Nation, patrimoine, 1789 - 1815, Ed NRF Gallimard - 1997, p 96. Le mot Musée est employé, par référence au fameux Muséon d'Alexandrie, ce cénacle de savants et d'érudits réunis à Alexandrie par la dynastie des Ptolémés, pour donner à produire et à discuter la science. C'est d'ailleurs pour l'essentiel la définition qu'en donne au XVIIIè l'encyclopédie de Diderot et d'Alembert.

12 le Belvédère du Vatican pour Pape Pie V vers 1580, le Palazzo Vecchio à Florence pour les Médicis, la Kunstkammer de Munich pour le duc Albrecht V de Bavière, le château d'Ambras près d'Innsbruck pour l'archiduc Ferdinand de Tyrol.

13 Schaer Roland, op. cit., p.27.

14 Raichvag Daniel, Jacques Jean, Savants et ignorants, une histoire de la vulgarisation, Ed Seuil, 1991, p.198. 
À partir de la fin du XVIIe, puis progressivement au XVIIIe, les cafés où " l'intelligence côtoie la noblesse" se développent dans le même esprit et rencontrent un vif succès. Ouverts à tous (il suffit de s'acquitter d'une consommation) ils affirment la dimension publique du débat. À la différence des salons, ils sont véritablement des lieux publics. Et les consommateurs y font l'apprentissage de la parole publique, polissent leurs arguments, élaborent leurs opinions, confrontent leurs points de vue, développent leur capacité de raisonnement.

Le phénomène de sociabilité cultivée constitutif de l'espace public va s'étendre à l'ensemble des domaines culturels et artistiques. À l'instar de la science, le théâtre, la musique et la peinture sont bientôt eux aussi dotés d'un public. A partir du moment où il assume tout ou partie des coûts de réalisation et où il devient non seulement consommateur mais usager, il est en mesure de porter un jugement critique ou esthétique sur les contenus. Dans son fameux tableau de Paris, Louis Sébastien Mercier, libre-penseur de cette fin de siècle, donne le ton : "Le mot Cour n'en impose plus comme au temps de Louis XV. On ne reçoit plus de la Cour les opinions régnantes, elle ne décide plus des réputations, en quelque genre que ce soit ; on ne dit plus avec une emphase ridicule, la Cour en a décidé ainsi. On dit nettement, elle n'y entend rien, elle n'est pas dans le point de vue... La Cour a donc perdu cet ascendant qu'elle avait sur les beaux-arts, sur les lettres et sur tout ce qui est de leur ressort", et de conclure, "c'est de la ville que part l'approbation ou l'improbation adoptée dans le royaume "

Habermas le décrit bien, au départ, la sphère publique bourgeoise est indécise, encore très imbriquée dans la sphère publique aristocratique dont la Cour était le support, et dont elle ne fait que copier les habitudes, les belles manières, les façons d'être. "L'avant-garde bourgeoise, issue des classes moyennes cultivées, assimile l'art du raisonnement au contact du "grand monde", d'une société aristocratique... qui de son côté... se détachait en effet toujours davantage de la Cour au profit de la ville où elle $\mathrm{y}$ formait un contrepoids "16. Les salons, puis les cafés, les réunions d'habitués constituent des espaces permissifs où des catégories sociales de plus en plus larges se côtoient, où la bourgeoisie apprend de la noblesse, avant de prendre son envol. Aristocrates humanistes et intellectuels bourgeois, à travers leurs conversations en société, " qui prirent aussitôt la forme d'une critique publique ", rompent, peu à peu, toutes les attaches qui les liaient encore à la Cour dont l'influence va en déclinant ${ }^{17}$.

\section{L'espace public médiatisé par la conversation et la publicité}

Quelles que soient leurs différences, insiste Habermas "salons et cafés dans l'étendue et la nature de leurs publics, dans leurs styles de vie, dans la tournure de leurs raisonnements et l'orientation de leurs principes, ne cessent (néanmoins) d'incarner une des aspirations à la discussion permanente entre personnes privées..."18. Le débat est la pierre d'angle de la théorie de l'espace public, il nourrit les conversations, mais, en même temps, doit satisfaire aux exigences de la raison. Le point de vue théorique repose là sur les travaux de Kant "Notre siècle est particulièrement le siècle de la critique à laquelle il faut que tous se soumettent" alors que "la religion par sa sainteté, et la législation par sa majesté veulent ordinairement s'y soustraire "19. Il esquisse l'exigence d'un espace public comme milieu où la

15 Mercier Louis Sébastien, Tableau de Paris, nouvelle Édition, Amsterdam 1782 - 1783 T. IV, p.261 - 264. Cité par Chartier Roger, Les Origines culturelles de la Révolution Française, Ed.du Seuil, 1990, p.217.

16 Habermas, op. cit., p.40.

17 Habermas, op. cit., p.41.

18 Habermas, op. cit., p.46.

19 Préface à la première édition de la critique de la raison pure, A XI. 
critique, l'usage libre de la raison, doit pouvoir se déployer, rencontrer d'autres critiques et progresser ainsi indéfiniment, aussi loin que possible. Une fois pénétrée dans l'espace public, la pensée doit "se développer", "se confirmer" jusqu'à " unir le suffrage de la réflexion ", qui ne s'imposera à tous que lorsque les personnes sont tombées d'accord "d'après l'idée qu'elles se font d'une pensée plus juste "20. De la sorte, progressivement, le public s'arroge le droit de mettre en débat des sujets d'intérêt général, culturels, politiques, sociaux, métaphysiques, qui jusque-là n'avaient jamais été mis en question, ou plutôt, dont la discussion était l'exclusivité du clergé et de l'État ${ }^{21}$.

Notons-le, Kant prône la liberté de pensée et d'expression pour une lente maturité du corps social. Il ne croit pas, pour cette raison, à un renversement brutal de la situation. " Un public ne peut accéder que lentement aux lumières. Une révolution entraînera peut-être le rejet du despotisme personnel et de l'oppression cupide et autoritaire, mais jamais une vraie réforme de la manière de penser; bien au contraire, de nouveaux préjugés tiendront en lisière, aussi bien que les anciens, la grande masse irréfléchie ${ }^{, 22}$.

Un autre élément fort caractérise la théorie d'Habermas, celui de la publicité faite aux débats par l'intermédiaire des nouveaux médias de l'époque. La discussion, les opinions, ne se propagent pas seulement de proche en proche, par personne interposée ; elles ont trouvé dans l'essor des moyens publics de communication écrite, un support et un relais privilégié. La poste nouvellement créée devient le média privilégié des salonniers. L'échange épistolaire leur permettait d'entretenir ainsi des discussions argumentées, dialectiques et suivies, sur les sujets les plus variés. Tandis que de leur coté les cafés privilégieront la presse naissante. Au contraire des revues de critique (plus distantes et plus sérieuses), les premiers hebdomadaires publiés sont directement partie prenante des discussions de cafés. "Les articles ne devinrent pas seulement l'objet des discussions du public au café, mais furent aussi compris comme leur organe... La même discussion se poursuivait transposée dans un autre média, afin de pouvoir pénétrer à nouveau, après avoir été lue, dans son milieu d'origine : la conversation "23.

Chartier conclut à " une véritable révolution de la lecture" dans la seconde moitié du XVIIIe siècle. Il écrit : "la croissance de la production du livre, la multiplication et la transformation des journaux, le succès des petits formats, l'abaissement du prix du livre... jouent à n'en pas douter un rôle essentiel dans les détachements critiques qui, partout en Europe, et particulièrement en France, éloignent les sujets de leur prince et les Chrétiens de leur église "24. Un nouveau rapport au texte s'est aussi construit, irrespectueux des autorités, tour à tour séduit et déçu par la nouveauté, et surtout, peu enclin à la croyance et à l'adhésion... " détaché des dépendances et des obéissances qui fondaient les représentations anciennes... "25

D'abord timidement éclairée par la culture et les arts, nourrie par la lecture, ayant appris à faire usage de sa raison, à forger ses opinions dans la discussion, la bourgeoisie en viendra à aborder des questions plus politiques et à revendiquer un rôle sur l'organisation de l'État.

\section{Espace public et médias de masse}

\footnotetext{
20 Kant Emmanuel, Réponse à la question : qu'est-ce que les lumières? Ed. La Pléiade, p.211, 214.

21 Idem p.47.

22 Idem p.211, 214

23 Habermas, op. cit. p.53.

24 Chartier Roger, Culture écrite et société, l'ordre du livre (XIV - XVIIIè siècle) Albin Michel 1996, p.32.

25 Idem p.114 et 115.
} 
Dans la dernière partie de son ouvrage, Habermas utilise l'idéal type d'espace public pour analyser la société contemporaine. Il s'intéresse à la façon dont l'espace public évolue et surtout, comment, dans la société contemporaine, il disparaît, en même temps que la transformation d'un public qui discutait la culture en un public qui la consomme et se contente de regarder à la TV des simulacres de Débat où une petite élite s'approprie la parole de tous.

Déjà, dans une relecture critique qu'il fait de son ouvrage trente ans plus tard, lors de sa réédition, Habermas nuance son point de vue en se demandant s'il peut exister, à l'instar des lieux de discussion publique du XVIIIè siècle, des espaces publics partiels susceptibles de représenter une alternative à l'hégémonie des médias de masse ${ }^{26}$. Il revient sur le sujet dans Droit et démocratie, l'un de ses derniers ouvrages, où il étudie cette capacité d'initiative de la société civile à révéler les dysfonctionnements du système, à soulever des problèmes qui ne sont pas traités, ou sont insuffisamment traités par les structures démocratiques, politiques ou administratives habituelles. Si historiquement l'espace public se forme dans les salons, les cafés, les lieux de spectacle et les publications; aujourd'hui, écrit-il, "c'est dans des constellations tout à fait différentes que la sphère d'une société civile vient d'être redécouverte "27 Et de citer les associations revendicatives, les syndicats, les mouvements sociaux, les groupements de consommateurs, les conseils de parents d'élèves, les lieux de rencontres informels, la rue, les bistrots, les institutions de la culture et de la communication qui forment l'espace public organisé, théâtres et salles de concert. On pense bien sûr aux musées, même s'il n'en parle pas. Il cite par contre les espaces publics de la vulgarisation scientifique, de la littérature, de l'art. Toutes ces organisations conclue-t-il ont en commun d'être ouvertes aux profanes et d'avoir la capacité de formuler des controverses publiques de plus en plus efficaces, si bien que toutes ensembles, elles constituent un tissu d'une grande complexité, une "multitude d'arènes" permanentes ou ponctuelles, matérielles ou virtuelles, aux " frontières poreuses ", "fluctuantes", se chevauchant les unes les autres. Elles sont d'une part, ouvertes à tous et notamment aux profanes, et d'autre part ouvertes sur l'extérieur et sur "l'arène centrale de l'espace public politique ". Elles font " figure de médiateur entre, d'un côté le système politique, et de l'autre, le secteur privé du monde vécu "28. On le voit, il ne s'agit pas seulement de groupes de pression, de mouvements revendicatifs orientés vers des objectifs précis, mais de l'ensemble des médiations par lesquelles la société civile participe aux débats politiques, se pense et se projette dans l'avenir. On pressent que les musées, et singulièrement les espaces de médiation scientifique, peuvent devenir un élément fort de ce nouvel espace public.

\section{2 - LES MUSÉES DE SCIENCE ET VULGARISATION AU XIX}

Contrairement au siècle des lumières libérateur, au XIXe s'opère une reprise en mains de l'espace public par la bourgeoisie qui s'affole de ne plus contrôler la vague révolutionnaire qui la porte au pouvoir ${ }^{29}$. Elle aspire à se distinguer de la masse et à remettre cette dernière à sa place. Désormais la culture savante deviendra son apanage, et comme elle l'avait été pour l'aristocratie le signe d'appartenance aux élites. Les musées joueront là un rôle important, et particulièrement les musées des sciences qui vont installer l'autorité des scientifiques et marginaliser ou faire cesser le débat partout ailleurs.

26 Habermas Jürgen, L'espace public, trente ans après, Quaderni $\mathrm{N}^{\circ}$ 18, automne 1992, p.162.

27 Idem p. 393.

28 Habermas Jurgen, Droit et démocratie, op. cit. p.401.

Voir : Poulot Dominique, Musée, nation, patrimoine : 1789-1815, Ed. Gallimard NRF, 1997. 
Le 10 juin 1793, le Jardin royal des plantes est transformé en Muséum d'histoire naturelle, " une sorte de métropole de toutes les sciences utiles à l'agriculture, au commerce et aux arts..." Des personnalités célèbres, établies, dont l'autorité est incontestée prennent, chacune en main, une discipline, Daubenton la minéralogie, Fourcroy la chimie générale, Lamark l'histoire naturelle des insectes, Desfontaine et Jussieu se partagent la botanique, il en est de même pour l'iconographie naturelle, la géologie, l'anatomie humaine, celle des animaux... Et Laissus de commenter, " les professeurs du muséum règnent sans partage sur les sciences de la nature et sur l'enseignement de celles-ci. Ils sont à de rares exceptions tous membres de première classe de l'institut, puis de l'académie des sciences. Beaucoup d'entre eux appartiennent à l'académie de médecine, Plusieurs enseignent au collège de France, à la faculté de médecine, à l'école centrale "30 .Le mouvement se poursuit en province, au point qu'à la fin du siècle il n'est pas de grande ville digne de ce nom en France, et en Europe, qui ne puisse s'enorgueillir d'avoir son musée d'histoire naturelle. Parallèlement, les sociétés savantes ont toutes été dissoutes en vertu de la loi Le Chapelier qui interdit toute forme d'association (elles se reconstitueront à partir de la seconde moitié du XIXe). De même, les cabinets de curiosités entachés de leurs origines aristocratiques et ecclésiastiques, disparaissent ou seront regroupés dans les musées.

Les musées des sciences deviennent l'élément clef d'un vaste projet scientifique, la systématique, faire un inventaire exhaustif du monde ${ }^{31}$. Il s'agit de rassembler en un ensemble ordonné d'échantillons les éléments composant le règne des trois ordres : minéral, végétal et animal de toute la terre; et d'envoyer des missions d'exploration dans tous les coins du globe pour qu'elles en ramènent des spécimens qui font ensemble la " nature terrestre ". Les musées ayant la mission de les conserver, de façon la plus immuable possible, jusqu'à construire un de ces fameux panoptiques concentrant en un lieu les objets de savoir, pour les donner à étudier aux savants ${ }^{32}$.

Encore faut-il adopter les mêmes règles du jeu pour rendre possible la comparaison entre tous et la cumulativité des travaux de chacun. À l'anarchie frissonnante des cabinets de curiosités, où chacun procédait à sa façon et en débattait avec les autres, il faut substituer un nouvel ordre, produire un langage spécialisé, précis et rigoureux, définir des conventions, uniformiser les méthodes, et surtout imposer à tous le même système de classement des collections (la taxinomie). Le poids de l'institution installée dans des bâtiments prestigieux, ostentatoires, confère aux professeurs des muséums l'autorité nécessaire pour faire cesser le débat partout ailleurs et imposer un seul point de vue, le leur.

La recherche devient affaire de professionnels et dans ce grand partage entre savants et ignorants, les amateurs éclairés sont exclus, renvoyés dans le camp des seconds.

\section{La vulgarisation, hors du musée fermé}

Les profanes ne sont autorisés à pénétrer dans le musée qu'à partir de la seconde moitié du XIXe, mais une demi-journée seulement par semaine, le dimanche après-midi. Les muséums imposent maintenant de nouveaux rapports entre savants et ignorants. Ces derniers ne sont plus tolérés que pour recevoir des leçons de vulgarisation. Dès qu'ils en ont la possibilité, les

30 Laissus Yves, Le muséum d'histoire naturelle, Ed. Gallimard, 1995, p.20 à 22 .

31 Héritier-Auger, Les musées de l'éducation nationale, Mission d'étude et de réflexion, Ed. La documentation Francaise, 1991, p. 36 et suivantes.

Van Praet Michel, Fromont Cécile, Eléments pour une histoire des Musées d'Histoire Naturelle en France, in Musée et recherche, Ed. OCIM 1995, p.60.

$32 \mathrm{Cf}$ : Rasse Paul, La recherche dans les musées, in Alliage $\mathrm{N}^{\circ} 25,1996$. 
conservateurs des grands musées y font construire un amphithéâtre à l'instar de ceux du muséum national de Paris où les scientifiques de l'établissement prennent l'habitude de donner des conférences qui ont un vif succès. Là on est loin des formes de sociabilité cultivée qu'entretenaient les cabinets de curiosité. La relation est cette fois celle du maître à l'élève. De fait, longtemps, les seuls visiteurs tolérés sont les scolaires, ou plutôt les étudiants encadrés par leurs professeurs ; sans doute curieux et attentifs, mais surtout disciplinés, studieux, silencieux, soumis, une attitude qui préfigure les nouveaux rapports au savoir.

À cette époque, la science se donne en public non seulement dans les grandes conférences de la Sorbonne ou de l'école polytechnique, mais aussi à l'occasion des expositions universelles, des foires et encore sur le lieu de travail, comme dans la gare de Bordeaux ou dans le quartier populaire de Belleville. Là, les médiateurs sont souvent animés d'un esprit d'éducation des masses dans la perspective de leur émancipation. Pour autant, il n'est plus question de participer à la constitution des connaissances savantes ou de contrôler leur développement.

On peut se demander si le fait d'avoir fait cesser le débat avec l'extérieur n'a pas contribué à fossiliser le débat à l'intérieur, au sein de la communauté scientifique ; le poids de l'institution muséale favorisant le dogmatisme et l'hégémonie des paradigmes en vigueur, jusqu'à vitrifier tout ferment de crise régénératrice.

De même que les musées des Beaux-arts, repliés sur leurs exigences académiques, étaient passés à côté de mouvements artistiques majeurs, les musées d'histoire naturelle sont passés à côté des révolutions survenues dans leurs champs disciplinaires : les théories de l'évolution d'abord, puis l'écologie. Ayant perdu leur aura scientifique, ils n'intéressaient plus personne. Le public qui n'y avait jamais été admis que pour s'y recueillir, soumis qu'il était à une liturgie qu'il ne comprenait pas, s'en désintéressa. Et Van Praet de conclure : " retranchés sur leur discipline d'excellence, en particulier la taxinomie, les musées d'histoire naturelle ont vécu jusqu'à la fin des années 1980 une telle période de torpeur, que la communauté scientifique, quand elle rechercha des vitrines culturelles de ses savoirs, se tourna vers d'autres institutions comme les Parcs Naturels, ou les Centres de Culture Scientifique ".

Depuis, on doit noter un regain d'intérêt pour l'institution muséale, et plus particulièrement pour les musées d'histoire naturelle. Bon nombre d'entre eux ont été rénovés ou sont en cour de rénovation. L'émergence du public en ces lieux désuets, abandonnés de tous, condamnés à la poussière et à l'oubli, a considérablement élargi les missions et modifié le fonctionnement des musées. La communication, desserrant l'étau de la recherche et de la conservation en a fait un espace de médiation culturelle sur la science. Les conservateurs des musées d'histoire naturelle en déshérence ont bien compris que la recherche leur échappait et ne justifiait plus leur existence que de façon marginale. Désormais, leur salut serait d'ordre culturel et mémoriel. Reste à préciser comment, s'agit il seulement de séduire les visiteurs potentiel avec des stratégies marketing offensives et le recours aux nouvelles technologies, mais de s'en tenir aux processus classiques de la vulgarisation, ou bien le musée peut-il aussi être un espace de débat sur les grand enjeux scientifiques de l'époque. Ici, le concept d'espace public nous permettra de faire une critique des actions engagées dans cette perspective et de mettre en évidence des axes de dépassement possible.

\section{3 - ESQUISSE DE L'ESPACE PUBLIC DANS LA MÉDIATION DES SCIENCES}

Quels sont les éléments forts qui structurent le concept d'espace public tel que nous nous sommes efforcés de le dégager? 
1/ L'espace public ne se constitue pas isolement, mais puise son énergie au sein de mutations économiques et sociales profondes qui travaillent la société, et à partir du XVIIe, l'essor de l'industrie et du commerce bouscule partout une société archaïque, artisanale et rurale figée par la tradition, les dogmes, l'ordre établi. Il conduit à l'émergence d'une nouvelle classe sociale avide de reconnaissances, encore profondément liées au peuple dont elle est issue, mais qui aspire à devenir, à participer au pouvoir. Au départ, on le voit bien, la bourgeoisie la plus opulente est admise à la cour puis dans les salons aristocratiques. Elle apprend, se mêle à la noblesse, espère encore y être assimilée, d'où sont investissement dans la culture, car elle sait bien, comme monsieur Jourdain, que là résident les clefs de son ascension sociale. Et puis elle doit bien se rendre à l'évidence au fur et à mesure qu'elle élargit sa base sociale qui elle n'a pas accès à la cour. Elle ne sera jamais l'aristocratie parce qu'elle est la masse, alors que l'aristocratie ne peut perdurer qu'à condition de rester une toute petite élite isolée par sa culture et des modes de vie la distinguant radicalement du peuple et légitimant sa domination.

La bourgeoisie découvre de nouveaux rapports à la culture par la discussion critique, l'usage de la raison, la publicité. Elle n'invente pas une nouvelle culture, radicalement différente, mais la génère à partir de la précédente qu'elle s'approprie et transforme progressivement, jusqu'à la faire sienne ; et paradoxalement, jusqu'à la faire différente dans son essence même. On le voit bien, ce n'est pas une armée qui entre en force ou une révolte sanglante de la plèbe, ni même la puissance d'une nouvelle idéologie qui enflammerait la foule, ce n'est qu'un long, lent, mais irréversible travail du corps social sur lui-même pour sa maturité

2/ Forcement, la venue en masse de la bourgeoisie dans le champ de la culture savante la transforme. Et si elle s'arroge le droit, au même titre que l'aristocratie et les princes, de mettre en discussion les savoirs savants, les valeurs esthétiques ou politiques du moment, elle partage les exigences du débat. Les discussions de salon, de club, ou de cafés des lumières ne sont pas les discussions de "café du commerce". Elles se nourrissent de l'ensemble des savoirs et des cultures du monde occidental de l'époque, elles sont avides d'informations couchées dans les livres et les revues, elles se confrontent aux réalisations artistiques passées ou contemporaines, elles intègrent les découvertes les plus récentes, elles se passionnent pour les controverses, les polémiques qui se nouent ailleurs et les rebattent dans leur propre cercle. Inversement, elles s'en font l'écho dans les correspondances épistolaires et les hebdomadaires pour qu'elles soient reprises dans les autres espaces de débat. Aux simplifications faciles de l'esprit, "l'honneste" citoyen opposent cette érudition du monde saisie dans sa complexité et sa diversité, cette exigence d'une polémique élargie par médias interposés à l'ensemble des autres débats, pour faire en sorte qu'il ne puisse jamais se refermer sur lui même et ses certitudes. La culture encyclopédique qui fait exister et caractérise l'homme des lumières, se construit rigoureusement par une incessante gymnastique de l'esprit, où alternent lectures, écritures, discussions, expérimentations scientifiques, expériences esthétiques, où le plaisir se gagne dans l'effort et le dépassement de soi.

L'idéal type d'espace public, nous allons maintenant l'utiliser pour analyser les mutations en cours dans le champ de la culture, et singulièrement, de la communication scientifique et technique (CST).

On peut commencer par resituer le mouvement dans un contexte lui aussi historique, où la classe moyenne s'est considérablement accrue et représente la majorité de la population des pays développés. Elle est la grande bénéficiaire de la croissance économique et de la société de consommation de l'après-guerre. Elle a obtenu un niveau de vie, de santé, de confort, incomparable. Par média de masse interposée, ses connaissances de l'histoire, de l'art, de l'actualité, atteignent, là aussi un niveau inégalé, jusque-là réservé aux élites les plus proches 
du pouvoir. Les barrières entre classes sociales n'étant plus aussi étanche, une fraction des couches moyenne peut espérer accéder à l'élite mondiale et cette aspiration constituer un puissant moteur. En même temps, là aussi il faut bien se rendre à l'évidence que cette possibilité n'est offerte qu'à une extrême minorité ; pour la majorité, les efforts de chacun pour s'élever ne le conduiront au mieux qu'à maintenir sa position sociale. Dès lors la question est de savoir si la classe moyenne peut s'inventer un destin collectif autre que celui que lui désignent les stratèges du marketing, de la communication et de la publicité. Peut-elle refuser de n'être que la somme des micro-décisions individuelles de consommateurs ou d'actionnaire ? D'autant plus qu'aujourd'hui, le système qui l'avait portée s'essouffle. Elle se sent menacée dans son confort et sa sécurité, dans son existence même, par la compétition économique, les revendications du tiers-monde, les exactions insécurisantes des laissés pour compte, mais aussi par le développement de la technoscience, la dégradation de l'environnement, les risques de cataclysmes écologiques.... Pour la première fois depuis longtemps, on prédit que les conditions d'existence de ses enfants seront probablement plus dures, en deçà de celles des parents. Pour rester compétitive, dans un univers qui se transforme de plus en plus vite, elle doit sans cesse innover, se dépasser, s'adapter, anticiper. Son avenir semble lui échapper, et en même temps les grandes idéologies messianiques ont perdu cette aura qui les faisait être des solutions de rechange prêtes à l'emploi.

Fragilisée, cette classe moyenne, dont nous sommes tous, hésite. Doit-elle continuer à se gaver de bien de consommation bon marché, s'abrutir d'analgésiques télévisuels, se défouler au spectacle de joutes sportives et de scènes de violence servies jusqu'à l'écœurement dans l'arène cathodique mondiale ? Peut-elle encore espérer se replier sur ses acquis et les défendre bec et ongles, doit elle s'abîmer dans la compassion pour les malheurs du monde, les frasques des petites princesses ou les victimes des catastrophes naturelles, la protection des animaux ou les misères des enfants persécutés, lui faudra-il se rendre à la révélation de nouveaux prophètes ? Peut elle reprendre la main et retrouver la maîtrise de son histoire, gagner du pouvoir en participant aux décisions qui façonnent en même temps que son avenir, son environnement scientifique et technique ? Enfin, peut-elle inventer de nouvelles voies au progrès en mettant en débat et en question les axes directeurs de la modernité ?

On peut lire en filigrane les prémices d'un tel projet, quand, dans la foulée des élections de 1981 et des assises nationales de la recherche, le gouvernement socialiste entreprend un ambitieux programme de développement de la CST (culture scientifique et technique), associant le ministère de la culture et de la recherche. Le Président du Conseil national de la culture scientifique, technique et industrielle, mis en place en 1985 pour coordonner les initiatives, déclarait, le jour de son intronisation en présence des Ministres de la culture et de la recherche : "Si nous voulons garder un sens au mot démocratie, il faudra bien que nous nous dotions des moyens, encore embryonnaires aujourd'hui, d'un choix démocratique sur les questions scientifiques et techniques qui, de plus en plus, conditionnent l'avenir de notre société, par exemple en matière de santé, de défense ou d'énergie "33.

En fait de "démocratie", les acteurs chargés de développer cet ambitieux programme sur le terrain se sont le plus souvent contentés de son avatar, "la démocratisation", c'est-à-dire, de rendre les lieux d'information scientifique accessibles à tous, de gagner des fractions de public qui étaient jusque là exclus de ces espaces là, dans une perspective non pas de citoyenneté

33 Lévy Leblond Jean-Marc, Une culture en mouvement, texte de l'allocution présentée le 10 septembre 84, devant les Ministres de la culture et de la recherche, texte cité in Mettre la science en culture, Ed. Anais 1986, p.33, voir aussi, Pour une politique de la culture scientifique et technique in le monde aujourd'hui du 28/28 mai 1984. 
mais d'éducation des masses. Il s'agissait en effet d'amener le peuple à fréquenter les musées pour pour qu'il puisse se rendre à la vérité des collections et de la parole savante. Et les Musées d'histoire naturelle, de développer des processus, souvent aussi sophistiqués que peu efficaces de vulgarisation, qui au contraire de permettre l'accès de tous au débat affirmait l'autorité des scientifiques.

Il faut commencer par noter le relatif échec des organisations qui ont mis en application ces conceptions avec le plus de conviction. Dix ans après les premières initiatives, le bilan est très mitigé. D'abord parce que le nombre de visiteurs reste limité et tend même à s'éroder, ensuite parce que ce sont un peu toujours les même catégories déjà cultivées qui fréquentent ces musées, tandis que les enquêtes d'évaluation réalisées à la sortie des expositions montrent que les apports didactiques sont bien pauvres. Faut-il en conclure que ce grand public est décidément trop inculte et rétif, ou bien, peut-on imaginer qu'en dehors des situations sociales contraintes, ou professionnelles obligées et rémunérées, les gens acceptent de moins en moins d'être cantonnés dans des rapports où ils sont mis en situation d'infériorité. Et s'ils font l'effort de se cultiver, c'est pour nourrir et construire leur vision du monde au sens actif et positif du terme, dans une perspective citoyenne.

Bref, le musée peut-il être un lieu où les gens viennent autant pour s'ouvrir aux vérités des autres que pour cultiver la leur, en conjuguant leur expérience et des savoirs acquis de l'extérieur. Dès lors ce qui les intéresse, c'est par exemple moins de comprendre le fonctionnement d'un atome ou d'un réacteur, que de réfléchir aux conditions de développement de ces techniques, à l'indépendance et au confort qu'elles nous apportent comme aux risques qu'elles font courir à tous, nous, nos enfants et futurs petits-enfants. Et dans cette perspective ils veulent s'informer, en débattre, donner leur opinion nourrir et complexifier leur point de vue. C'est l'hypothèse que nous formons et dont on peut repérer ça et là les prémisses ; le musée tendrait à devenir un de ces fameux " espaces publics partiels" dont Habermas décèle l'émergence.

1. Sur la forme, nous verrons que les interactifs ont contribué activement à désacraliser l'espace muséal.

2. Sur le fond, nous nous intéresserons aux thèmes des expositions qui portent de plus en plus fréquemment sur des sujets à controverse, mêlant la technoscience et la société, notamment, dans le domaine sensible de l'environnement.

3. Au plan de la réception enfin, nous aborderons cette découverte récente, que les visites se font en groupe et l'exposition s'offre aux visiteurs comme espace de discussion et de débat collectif.

\section{Les interactifs, pour désacraliser l'espace muséal}

Confrontés aux limites de la vulgarisation, à la recherche de solutions nouvelles pour vaincre la réticence du public, les muséographes ne pouvaient qu'être séduits par les concepts d'interactivité s'inspirant des principes d'éducation informelle développés dans les pays anglo-saxons. Le projet repose, subrepticement sur l'idée que l'on peut apprendre sans effort, en s'amusant, en s'étonnant, en étant curieux, en manipulant des objets ou des images en fonction de ses intuitions et de ses envies du moment.

L'utilisateur ne sait pas ce qu'il cherche, il se promène dans le champ des savoirs au gré des stimulations et des possibilités offertes par le dispositif multimédia (autrefois des bornes informatiques, maintenant des $\mathrm{CD}$ rom). Les acquis à l'issue de la visite sont très différents, selon que le visiteur a des questions précises et une culture générale du sujet, ou qu'il surf sur le champ des sciences et glane ici et là des informations ponctuelles, parcellisées, sur des 
sujets qu'il ne maîtrise pas. Dans tous les cas, il n'est cependant déjà plus suspendu aux lèvres d'un conférencier, ni soumis à la lecture d'un savoir linéaire décliné dans l'exposition, il jouit à cet égard d'une relative liberté. Quant à la forme, le visiteur, n'est plus ce corps passif, soumis dans une attitude respectueuse que les conservateurs se sont efforcés de discipliner durant tout le XIXe. De ce point de vue, le musée perd sa dimension sacrée et le public expérimente de nouvelles attitudes plus actives à l'égard de ce qui est exposé, et des savoirs. De là à ce qu'il en vienne à mettre en question le contenu même des messages, il y a encore un pas à faire. Et ce pas il semble bien être disposé à le faire dans les domaines de l'environnement, et plus généralement chaque fois que l'on aborde des sujets touchant à son avenir, chaque fois que le développement des sciences devient problème de société.

\section{Controverses environnementales dans les musées de science}

Les musées d'histoire naturelle les plus dynamiques ont mis à profit l'inquiétude que suscite, un peu partout, la dégradation de l'environnement pour gagner de nouveaux publics et renouer avec les préoccupations sociales et politiques du moment. En effet, leurs collections et leur expérience de la taxinomie les prédisposaient à entreprendre des actions d'information sur la faune et la flore, mieux, sur la biodiversité et les théories de l'évolution, ou encore, sur les écosystèmes.

En fait, en matière d'environnement, l'institution muséale hésite encore. Doit-elle se contenter de faire de l'information scientifique, de donner et de défendre le point de vue savant, d'éduquer le public à l'écologie ? Inversement, le musée peut-il réellement devenir un lieu de débat sur la protection de l'environnement saisie dans la complexité des problèmes que pose sa défense ? Bien sûr, bon nombre de conservateurs n'y voient encore qu'une occasion, parmi d'autres, de redorer leur enseigne à bon compte, et sous prétexte d'éducation du public, de réduire le vaste débat que suscite la protection de la nature aux seules conceptions d'une poignée de scientifiques, si compétents et si sûrs de leur bon droit soient-ils. Les musées peuvent-il au contraire devenir un lieu de débat et s'engager dans l'élaboration d'une opinion publique susceptible d'influencer le cours des choses, d'inventer des solutions originales en complexifiant la perception des situations, en amenant les différents partis à prendre en considération le point de vue de l'autre ${ }^{34}$

Loin de courir après une neutralité qui risque le plus souvent de le conduire à évacuer tout ce qui fait problème, et donc à ne traiter que des sujets insipides, le musée peut-il s'engager, devenir un outil de communication et d'information du public sur des controverses ? Il renonce, alors, à asséner une vérité intangible, mais avance des arguments, qui ne sont que des arguments à porter au débat. Ce faisant, il participe à la formation d'une opinion consensuelle, pour autant qu'aucun autre argument ne vienne relancer le débat.

À la différence des conceptions antérieures où il s'agissait à tout prix de faire passer un message, et où les évaluations visaient à s'assurer de sa bonne réception, on trouve maintenant légitime que les visiteurs puissent avoir une demande. Ils ne sont plus seulement placés au centre des problématiques muséographiques comme récepteurs, mais aussi comme acteurs ; leurs questions, leurs inquiétudes sont étudiées et prises en considération ${ }^{35}$. Ainsi découvre-t-on que la science les intéresse comme fait sociétal, dans sa dynamique et dans la perspective d'une meilleure maîtrise de leur avenir.

34 voir: Davallon Jean, Grandmont Gerald et Schiele Bernard, L’environnement entre au musée, Ed.PUL, 1992

35 Le Marec Joëlle, Repenser la relation du musée à son public, in la révolution de la muséologie des sciences, Ed. PUL, 1998, P. 395.

Le Marec Joëlle, L'interactivité, rencontre entre visiteurs et concepteurs, Publics et musées N³, Ed. PUL, 1993, p.93. 


\section{Le visiteur collectif et le débat}

Après l'interactivité et le choix de sujets propices à la controverse, la troisième ligne d'avancée vers l'émergence d'un nouvel espace public dans la muséographie des sciences est à rechercher dans ce qui fait l'une des spécificités de l'exposition, celle d'être "un média froid". Elle s'offre naturellement à la distanciation. Le parcours du visiteur, même quand il est prévu et organisé par les muséographes, laisse à celui-ci la possibilité d'aller à son rythme, d'avancer, de revenir, de faire le tour, de s'arrêter, de se rapprocher, de s'éloigner, de repartir, de faire sa propre visite au gré des envies du moment, du temps dont il dispose, des gens avec lesquels il partage l'expérience. Car l'exposition est d'abord un espace d'interaction sociale entre les membres d'une famille, d'un groupe d'amis, d'un couple, qui ont décidé de faire la visite ensemble, mais aussi avec les autres visiteurs, qui sont là eux aussi au même moment ; et tous de s'observer, d'apprendre les uns des autres. Chaque groupe génère sa propre dynamique, qui fait la dynamique propre à chaque visite, en fonction de ce qu'il est, des expériences qu'il réunit, des liens qu'il tisse, des discussions qu'il provoque, de ce matériel social qu'il réactualise et enrichit.

La communication s'impose ainsi comme condition d'accès à des informations sensibles, écrit Bougnoux, et plus loin d'ajouter : " la communication est la politesse de l'information "36. La visite en groupes chaleureux, solidaires, conviviaux, facilite la mise à distance des messages d'information contenus dans l'exposition. Le groupe met les visiteurs en situation d'engager un dialogue à partir de ce qu'ils sont, avec ce qu'ils découvrent, avec ce qu'ils s'efforcent de comprendre des messages et même des intentions des muséographes. Et chacun négocie son rapport à l'exposition en fonction de ce qu'il sait déjà du sujet, de par sa formation, de par les autres médias d'information, de par son expérience vécue de la science et de la technologie.

Pour tout cela, l'exposition est un espace public privilégié pour la discussion, il devient un lieu de controverse nous dit Macdonald, où le public, ajoute Davallon " manifeste son goût et son opinion, en venant, ou ne venant pas, en critiquant ou non "37. Peu importe encore, à ce stade, de connaître le contenu de ces débats (même s'il mériterait d'être étudié), car l'essentiel n'est pas là. Il est de même nature que ce que disait Chartier de l'édition au XVII multiplication des publications a brisé le rapport de soumission à l'écrit, à l'autorité que représentaient les textes publiés. Là aussi, se joue une certaine forme d'émancipation culturelle, qui touche moins au contenu qu'au rapport du visiteur à ces contenus. En effet, le corps des visiteurs n'est plus discipliné, silencieux, dans une attitude de soumission aux principes d'éducation, il admire, apprend, chahute, s'étonne, joue, s'émeut, se délecte, se sent valorisé, mais surtout se découvre en capacité de discuter les choix muséographiques et les intentions des concepteurs, d'avoir une opinion personnelle ou collective sur la qualité de l'exposition et le discours des scientifiques, de l'argumenter et de la faire savoir autour de lui ${ }^{38}$.

Le musée deviendrait une agora opposant aux facilités de l'esprit, les exigences d'un débat public mûri aux contacts des témoignages de l'histoire et des sciences contemporaines, à l'articulation du passé et de l'avenir, un lieu symbolique, fort, de la cité, où se construit et se

36 Bougnoux Daniel, La communication contre l'information, Ed Hachette, 1995, p.99 et 135

37 Macdonald sharon, Un nouveau "corps des visiteurs" : musées et changements culturels, Publics et musées n'3, 1993, p.21

Davallon Jean, L'évolution du rôle des musées, Lettre de L'OCIM N 49, 1997, p.7. 
donne à entendre l'opinion publique à l'égard des grands et petits problèmes de société touchant à la science, à la technique et au progrès. Mais cette histoire-là est encore à inventer et à réaliser, car attention! il ne s'agit là encore, pour l'essentiel, que d'un infléchissement des activités des centres de sciences dont les professionnels n'ont parfois même pas encore conscience. Et les expériences innovantes se heurtent aux habitudes et aux recettes anciennes. L'idée de faire aussi du musée un espace public partiel (au sens habermatien du terme) doit être perçue comme un projet. Nous nous sommes efforcés de le formuler, d'en monter les fondements, la pertinence, l'actualité, mais il appartient aux acteurs sur le terrain d'en inventer les voies, de l'utiliser pour orienter leurs réalisations et leurs donner du sens. 